\title{
Typicité sensorielle de vins de l'AOC Coteaux du Layon et acceptabilité par les acteurs (professionnels et consommateurs) de vins avec des titres d'alcool volumique plus faibles / Sensory typicity of the PDO Coteaux du Layon: Acceptability by the actors (professionals and consumers) of wines with lower alcohol content
}

\author{
Cécile Coulon-Leroy ${ }^{1}$, Ronan Symoneaux ${ }^{1}$, Philippe Chretien $^{2}$, Aurélie Perrin ${ }^{1}$, et René Siret ${ }^{1}$ \\ ${ }^{1}$ USC 1422 GRAPPE, INRA, Ecole Supérieure d'Agricultures, Univ. Bretagne Loire, SFR 4207 QUASAV, 55 rue Rabelais, Angers, \\ France \\ ${ }^{2}$ Institut Français de la Vigne et du vin, Pôle Val de Loire, Beaucouzé, France
}

\begin{abstract}
In order to maintain an optimum sugar / acid balance in a changing context, winegrowers of the Coteaux du Layon PDO, a sweet wine of the middle Loire valley, wish to know if a final alcohol content (TAV) lower than the minimum imposed by the regulation of the PDO (11\% vol. today) would affect the typicality of the wines. To answer this question, a study was carried out in 4 steps. 1) To know the expected typicality of these wines, focus groups were conducted with 26 professionals. 2) In order to assess the acceptability of lower TAV wines, based on a sample of 24 wines (commercial wines and experimental wines with TAV between $8 \%$ vol. and $10 \%$ vol.), 31 professionals scored levels of exemplarity. 3) A trained panel made a fine sensory description. 4) Finally, a selection of 8 wines from these 24 were evaluated by 163 consumers, they scored levels of preference. The results of steps 1) and 2) show a strong consensus among professionals to assess the exemplarity of wines. Experimental wines obtained exemplary scores above the average of the scale and can therefore be considered as good examples. In step 3), the trained panel generated the sensory attributes to describe the wines: 21 descriptors of odors and aromas and 6 of flavors, which reveal the sensory complexity of the wines. In stage 4), in general, the 8 wines evaluated by the consumers were all appreciated. The results suggest that a sufficiently high amount of residual sugar content would enhance the acceptability of lower TAV wines by both professionals and consumers. The winegrowers of the syndicate of Coteaux du Layon PDO thus have factual elements to update the specification of the PDO (decreasing the minimum TAV threshold), in interaction with INAO (the French national institute of origin and quality).
\end{abstract}

\section{Introduction}

Les producteurs de vins de l'AOC Coteaux du Layon sont soumis à un contexte changeant qui les amène à repenser le profil analytique attendu de certains de leurs vins.

\subsection{Une concurrence internationale forte}

Les vins sous signes de qualité, et notamment les AOC, contribuent fortement à l'économie française en étant le second secteur d'exportation excédentaire après l'aéronautique ou en amenant 10 millions d'oenotouristes en France [1]. Cette production permet le maintien des territoires et paysages et une valorisation satisfaisante pour les vignerons puisqu'ils se basent alors sur une logique de différenciation [2]. Toutefois la concurrence est très présente à l'international avec de plus en plus de zones viticoles revendiquant un lien au terroir.

La revendication d'un lien à l'origine pour les vins, n'est plus une exclusivité des pays producteurs de «l'ancien monde». La concurrence internationale est de plus en plus forte, les vignobles français doivent affirmer leur identité : la typicité, celle-ci est liée à leur origine : le terroir. Les vins de l'AOC Coteaux du Layon n'échappent pas à cette concurrence internationale et à la nécessité de réaffirmer et valoriser leur typicité. Des travaux sur la typicité et le lien avec le terroir ont déjà été entrepris à l'échelle des AOC [3].

\subsection{Une production très soumise aux aléas climatiques et à Botrytis cinerea}

La récolte des raisins destinés à la production de liquoreux est soumise de façon importante aux aléas météorologiques, de plus en plus nombreux. Des pluies trop importantes en automne favorisent le développement de Botrytis cinerea sous sa forme pourriture grise qui peut affecter significativement le rendement et la qualité des raisins et ne pas permettre alors d'atteindre, naturellement, le minimum de sucre requis par le cahier des charges de l'AOC. La chaptalisation pour atteindre, si besoin, ce minimum requis, tend, par ailleurs, à être de plus en plus abandonnée. 
La forme pourriture noble de Botrytis cinerea, quant à elle, produit un antibiotique, la botryticine, qui tend à arrêter naturellement les fermentations. Cela se produit parfois avant le taux minimum d'alcool requis. Il est alors nécessaire de relancer artificiellement la fermentation même si l'équilibre du vin est satisfaisant pour attendre le minimum d'alcool réglementaire.

\subsection{Une production actuelle difficilement durable}

Selon l'indice de Huglin, la région viticole du Val de Loire a évolué d'un climat frais à un climat tempéré, avec une évolution de $+1.6^{\circ} \mathrm{C}$ en 50 ans [4]. Ce réchauffement a conduit à une avancée des stades phénologiques et à une évolution importante de la composition des baies. Une diminution de l'acidité des raisins est aussi constatée. Les vins doivent alors tendre à être un peu moins sucrés pour maintenir des équilibres sucres / acides optimaux.

Les tries successives nécessaires pour produire des liquoreux concentrés en sucre, couplés aux faibles rendements, amènent, de plus, à des coûts de production élevés et non rentables pour certaines exploitations.

Par ailleurs, les consommateurs tendent à préférer des produits plus faciles à boire pour une consommation plus fréquente, contrairement aux vins AOC Coteaux du Layon historiquement très concentrés et réservés à quelques occasions particulières.

\subsection{Une étude de la typicité des vins de l'AOC Coteaux du Layon pour évoluer vers une segmentation des vins}

Une des dimensions clés de la qualité d'un vin AOC est sa «typicité», qui est liée à son origine géographique, son cépage et les techniques de vinification [5]. Elle correspond à la propriété d'appartenance à un type, la typicité liée au terroir est une construction particulière qui concrétise l'effet du terroir pour un produit donné [6]. Elle peut être associée aux valeurs d'origine, d'authenticité et de tradition auxquelles sont de plus en en plus attachés certains consommateurs.

Dans le cadre de ce projet, le focus a été fait sur la typicité sensorielle et non sur la typicité au sens large. La typicité peut être théorique [7] suivant l'appartenance à une $\mathrm{AOC}$; conceptuelle suivant l'image que se font les connaisseurs du produit ou perceptuelle suite à une dégustation [3]. Cette dernière peut être qualifiée de typicité sensorielle.

Les vignerons de l'AOC des Coteaux du Layon considèrent que l'adaptation au contexte changeant passe par une segmentation des produits :

- Des vins concentrés et riches, avec des arômes propres aux vendanges tardives, avec un travail important du vigneron (plusieurs tris, sélection de cuvée) avec un prix de valorisation plus important que la moyenne de l'AOC. Ces vins sont notamment produits par les vignerons proches de la source du Layon, au sud du département qui ne bénéficie pas de parcelles sur les zones des AOC Coteaux du Layon Villages, Chaume ou Bonnezeaux.

- Des vins plus légers et vifs avec des arômes de fruits frais, aux coûts de production inférieurs, le prix de valorisation étant plus faible que la précédente catégorie. Ces vins permettent une part de risque plus faible (une fois les maturités avancées, il est difficile de «revenir en arrière» en cas d'aléas), facteur essentiel pour une pérennité des exploitations. Ils sont par ailleurs de plus en plus demandés par les clients. Les raisins sont récoltés à un titre d'alcool potientiel (TAP) plus faible.

Pour cette seconde catégorie, les vignerons de l'AOC Coteaux du Layon, souhaitent savoir si, pour ces cuvées à plus faible TAP, un TAV (Titre Alcoométrique Volumique) plus faible que le minimum imposé par la réglementation du cahier des charges de l'AOC (qui est de $11 \%$ vol. aujourd'hui) affecterait la typicité des vins.

\section{Matériel et méthode}

L'objectif global des travaux présentés visait à comprendre comment les professionnels de la filière et les consommateurs acceptent des vins de l'AOC Coteaux du Layon ayant un équilibre 'alcool acquis / sucres résiduels' ne correspondant pas à celui actuellement autorisé par le cahier des charges de l'AOC. Il s'agissait de vérifier si ces nouveaux vins candidats à l'AOC étaient définis tout de même comme de bons exemples de vins AOC Coteaux du Layon par les professionnels de la filière, si leurs caractéristiques sensorielles correspondaient à celles qui était attendues et s'ils n'étaient pas rejetés par les consommateurs. L'étude a été menée entre 2015 et 2017.

\subsection{Les vins de l'étude}

La sélection des vins de l'étude avait pour objectif de couvrir l'ensemble des profils sensoriels des vins de l'AOC Coteaux du Layon mais se trouvant dans les limites basses du cahier des charges en termes de taux d'alcool. La sélection a été réalisée par le Syndicat de l'AOC Coteaux du Layon. Les critères se sont portés sur des caractéristiques analytiques et techniques (TAV, quantité de sucres résiduels, millésime) et aromatiques. 16 vins commerciaux ont été sélectionnés : 14 de l'AOC Coteaux du Layon, notés «Layon» et 2 hors de l'AOC Coteaux du Layon, mais ayant des caractéristiques proches (un vin blanc de France et un de l'AOC Anjou blanc) notés «NonLayon». L'ensemble des vins sont exclusivement issus du cépage Chenin.

En complément, 8 vins expérimentaux ont été élaborés par l'IFV. Ces vins, notés «Expé», ont un TAV inférieur à ce qui est actuellement autorisé dans le cahier des charges. Ces vins ont été élaborés à partir de raisins de Chenin récoltés dans l'aire de l'AOC Coteaux du Layon avec une concentration en sucre moins importante. Certains vins ont été dilués à l'eau distillée pour atteindre certains TAV fixés.

Dans un second temps, une sous-sélection de 8 vins, 4 vins commerciaux et 4 vins expérimentaux, a été réalisée sur la base des caractérisations sensorielles des 24 vins faites par le panel entrainé et les professionnels. Ces vins ont été évalués par les consommateurs et ont servis de supports de discussion avec les professionnels lors de tables-rondes. Les critères de sélection des 8 vins ont été d'éliminer les vins avec défauts (comme un goût de bouchon), de conserver une large diversité de produits, de focaliser plus strictement sur les limites basses du cahier des charges au niveau du taux d'alcool et de 
conserver une répartition équilibrée de vins commerciaux et expérimentaux.

\subsection{L'évaluation de la typicité par les connaisseurs et porteurs de mémoire de l'AOC Coteaux du Layon}

La typicité sensorielle conceptuelle a été évaluée lors de tables-rondes par 26 professionnels de l'AOC Coteaux du Layon (producteurs, œnologues, techniciens et personnes formées aux contrôles organoleptiques).

La typicité sensorielle perceptuelle a été étudiée via la notion d'exemplarité lors de dégustations. Cette méthode a été mise au point par Ballester et al. [8] et utilisée par la suite par plusieurs auteurs $[7,9,10]$. Dans ce cas, l'échelle d'évaluation va de «mauvais exemple» à «bon exemple»

Le test d'exemplarité, sur les 24 vins, a été réalisé avec 31 professionnels de la filière viticole de la région Anjou, connaisseurs des vins de l'AOC Coteaux du Layon dont 18 producteurs, 9 œnologues, 1 ingénieur viticole et 3 personnes formées aux contrôles organoleptiques (25 hommes et 6 femmes, âge moyen de 46 ans). L'objectif était ici de d'évaluer le niveau d'exemplarité des vins de l'étude, d'identifier s'il existait un consensus concernant la typicité des vins AOC Coteaux du Layon et également d'évaluer les caractéristiques qui permettent de les identifier.

Une Analyse en Composantes Principales (ACP) normée aves les juges comme variables et les vins comme individus a été réalisée pour évaluer le consensus entre les juges. La typicité moyenne a également été projetée en variable supplémentaire.

Les professionnels ont ainsi été mobilisés en deux temps : dans un premier temps, lors de dégustation pour réaliser le test d'exemplarité et évaluer les caractéristiques sensorielles des 24 vins; et dans un second temps, pour participer à des tables-rondes couplant des discussions et des dégustations des 8 vins. Ces deux approches complémentaires ont permis d'obtenir d'une part des informations quantitatives, et d'autre part des informations plus qualitatives.

\subsection{La caractérisation sensorielle des vins par un panel entrainé}

Le profil conventionnel a été choisi comme étant la méthode la plus appropriée pour l'étude. En effet, une description précise des 24 vins était requise pour pouvoir les différencier. Le panel était composé de 15 personnes âgées de 42 à 65 ans, dont 9 femmes et 6 hommes. Les séances d'évaluation ont eu lieu au sein de la salle d'analyse sensorielle de l'USC Grappe, INRA, ESA. Cette salle est constituée de 20 box standardisés selon la norme AFNOR NF09-105 et équipée du logiciel FIZZ. Le panel a été formé à l'évaluation des vins de l'AOC Coteaux du Layon en s'entrainant sur des références olfactives et gustatives spécifiques aux moelleux et liquoreux.

Dans un premier temps la liste des descripteurs sensoriels a été établie à partir de la littérature existante sur les liquoreux et les vins de l'AOC Coteaux du Layon. Cette liste a ensuite été ajustée par le panel, par consensus lors des entrainements réalisés sur les vins de l'étude. La liste finale contenait 50 termes dont 6 descripteurs de saveurs ou
Tableau 1. Liste des 50 descripteurs sélectionnés par le panel entrainé et $\mathrm{P}$-value associées pour les descripteurs discriminants $(>10 \%)$.

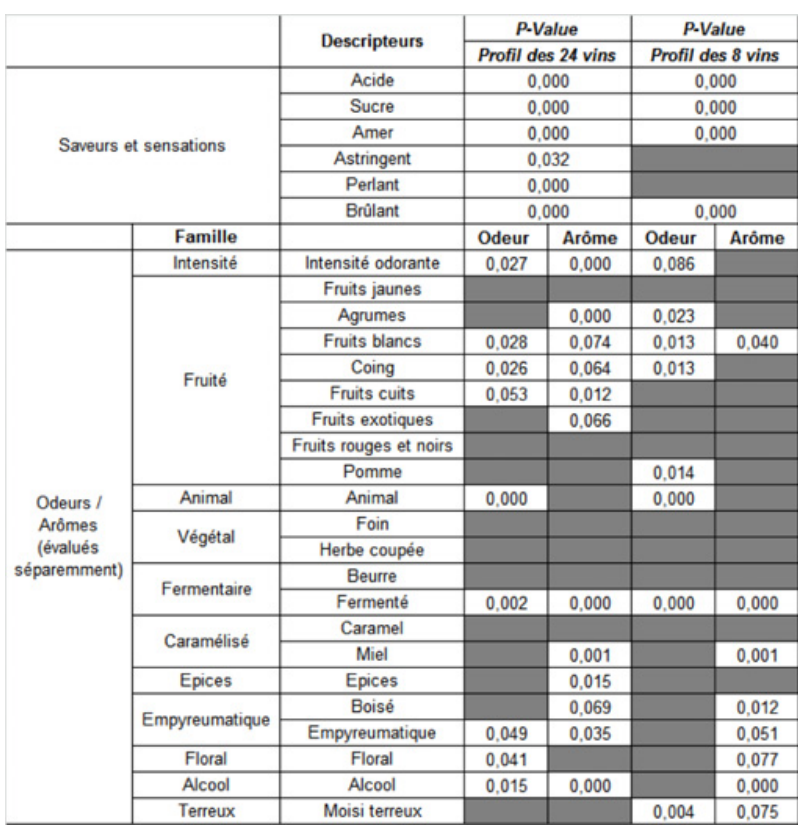

sensations et 22 couples d'arômes et odeurs (Tableau 1). Un entrainement spécifique à la reconnaissance de ces descripteurs a ensuite été réalisé.

Des analyses de la variance (ANOVA) ont été réalisées avec un modèle mixte à trois facteurs. Le facteur «juge» est considéré comme un effet aléatoire, les facteurs «produit» et «répétition» comme des effets fixes. Le modèle prend en compte les interactions entre les facteurs.

Une ACP a été réalisée à partir des descripteurs sensoriels significatifs à $10 \%$ afin d'obtenir une représentation multidimensionnelle des produits en résumant la variabilité entre les vins. Le tableau des moyennes sur le panel a été utilisé et les données ont été normées.

Les 24 vins ont été regroupés par une Classification Ascendante Hiérarchique en prenant en compte l'ensemble des dimensions de l'ACP, six groupes ont été pris en compte pour réaliser la sélection des 8 vins évalués par les consommateurs.

\subsection{L'évaluation de l'acceptabilité des consommateurs}

L'objectif de cette phase de l'étude était d'obtenir la perception des consommateurs sur les vins. Un test hédonique a été réalisé sur la sous-sélection de 8 vins afin de connaitre leur appréciation. Chaque consommateur devait noter le produit sur une échelle discontinue à 9 points allant de «extrêmement désagréable» à «extrêmement agréable». Il leur était également demandé de s'exprimer sur les qualités et défauts de chaque vin.

Ces tests ont été réalisés auprès de 163 consommateurs, à Angers et Lille, avec un nombre d'individus équilibré selon 3 critères : sexe, âge et ville. Le test s'est déroulé selon le même protocole dans les deux villes, dans des salles d'analyse sensorielle équipées du logiciel FIZZ.

Les vins ont été présentés aux consommateurs de façon monadique séquentielle et selon un plan d'expérience basé sur un carré latin de Williams. 


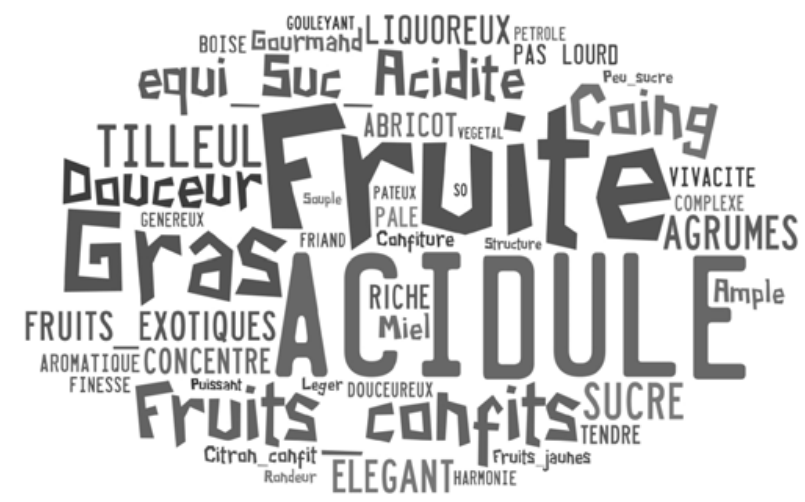

Figure 1. Citations des mots utilisés individuellement par les 26 professionnels, lors des tables-rondes, pour décrire un vin d'AOC Coteaux du Layon (la taille est proportionnelle à la fréquence de citation).

\section{Résultats et discussion}

\subsection{La typicité conceptuelle des vins de l'AOC Coteaux du Layon par les professionnels}

Le cahier des charges en vigueur (BO 26 juin 2014) indique : «Les vins sont racés et élégants. En bouche, ils allient la suavité à la frâ̂cheur, la puissance à la finesse et leurs arômes sont complexes. Aptes à un vieillissement prolongé, les vins font le bonheur des dégustateurs avertis». Les vins sont également décrits par l'interprofession comme ayant «une robe claire à profonde, jaune or aux reflets verts, évoluant vers un vieil or aux reflets ambrés ; le nez à des arômes de miel, acacia, fleur, citronnelle, ananas, abricot, coing, fruits frais pour les vins jeunes et notes de fruits confits sur les vins concentrés ou plus évolués ; sa bouche est ronde, onctueuse, équilibrée, franche avec de la fraicheur et vivacité en finale».

Lors des tables-rondes, les Coteaux du Layon ont été décrits individuellement par les 26 professionnels présents au total avec les 3 séances. Les 8 descripteurs qui ont eu les fréquences de citation les plus élevées $(>20 \%)$ pour caractériser un vin de l'AOC Coteaux du Layon sont : «fruité» (26 citations), «acidulé» (14 citations), «fraicheur» (12 citations), «moelleux» (9 citations), «gras» (9 citations), «équilibré» (8 citations), «fruits confits» (7 citations) et «jaune or» (7 citations). On retrouve quelques descripteurs du cahier des charges. Ces descriptions sont à relier à la notion de typicité conceptuelle [3].

Les professionnels ajoutent lors des discussions que les vins de l'AOC Coteaux du Layon les plus légers sont reconnaissables à leurs arômes de fruits frais alors que les notes de fruits secs apparaissent, elles, avec des vins plus concentrés. Les professionnels expliquent que les vins AOC Coteaux du Layon sont caractérisés par une certaine vivacité, apportée par le cépage Chenin qui les différencie par rapport aux autres liquoreux. D'ailleurs, l'équilibre est un mot fréquemment cité et notamment l'équilibre entre la quantité de sucre et l'acidité. Les termes «moelleux», «gras» sont également des mots qui reviennent fréquemment. Au niveau aromatique, ce sont des vins pouvant présenter des notes florales, et dont la couleur est jaune/dorée.

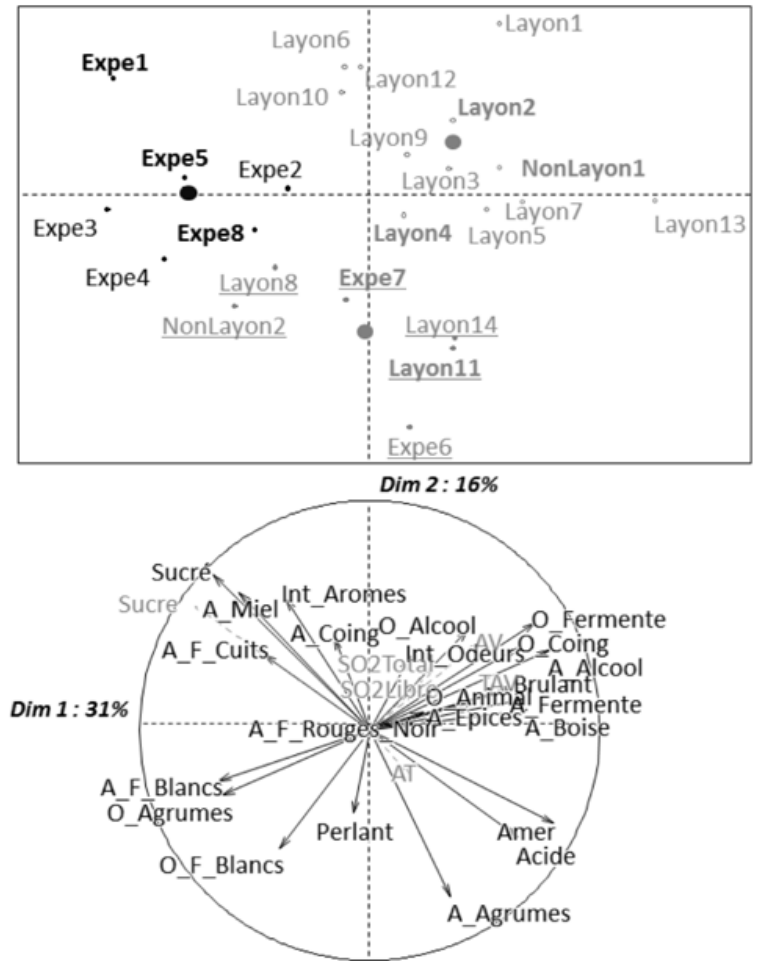

Figure 2. Analyse en Composantes Principales réalisés sur les 24 vins (Expe : vins expérimentaux) et 27 descripteurs discriminants les vins (au risque d'erreur de $10 \%, \mathrm{~F}$ : fruits, Int : intensité). Les variables en bleu n'ont pas été prises en compte dans l'analyse (AT : acidité totale, AV : acidité volatile). La première composante (abscisse) représente $33 \%$ de l'information et la seconde (ordonné) $15 \%$. Les groupes en noir, gris et gris souligné correspondent aux groupes optimaux obtenus suite à une Classification Ascendante Hiérarchique (critère de Ward).

\subsection{Les caractéristiques sensorielles des vins de l'AOC Coteaux du Layon par le panel entrainé}

Sur les 50 descripteurs utilisés, 27 permettent de différencier les 24 vins de l'étude (Tableau 1).

Un groupe est uniquement constitué de vins expérimentaux (à gauche de la Fig. 2). Ces 6 vins se caractérisent par une saveur et une quantité de sucre élevée, ainsi qu'un arôme de «miel» et une odeur de «fruits blancs». Ces vins sont aussi peu alcoolisés. Le groupe en bas du graphique Fig. 2 regroupe 2 vins expérimentaux ainsi que 4 vins commerciaux. Ils se différencient des autres par leurs arômes «agrumes» et une faible saveur «sucré». Enfin, le groupe de droite est composée de 12 vins commerciaux dont un des vins commerciaux qui n'appartient pas à l'AOC. Ces vins se caractérisent par un arôme et une odeur «alcool» qui est associé à un TAV élevé, mais aussi des odeurs «animal» et «coing». De plus, ils présentent une odeur et un arôme «fermenté».

Les 8 vins évalués par les consommateurs ont été à évalués par le panel entrainé au même moment (une année s'étant écoulée, il était nécessaire de revérifier les caractéristiques sensorielles). 6 descripteurs ne sont pas discriminants ni pour le profil des 24 vins, ni pour celui des 8 vins ; avec un taux d'erreur de $10 \%$ (Tableau 1). Globalement les caractérisations sensorielles sont proches. 


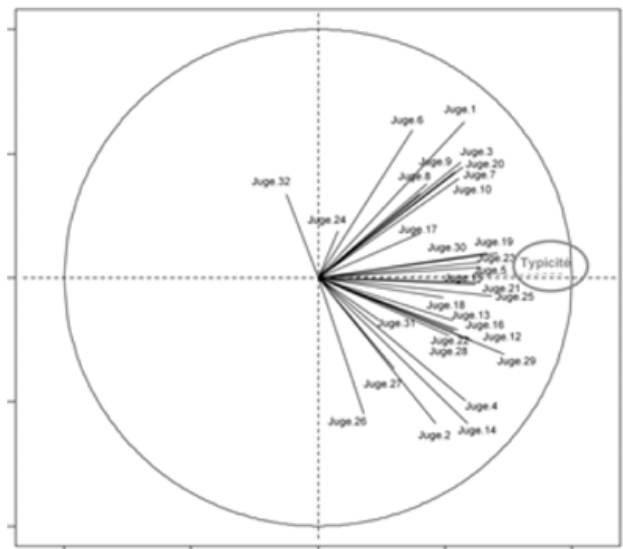

Figure 3. Analyse en Composantes Principales sur les notes d'exemplarité des professionnels. La première composante (abscisse) représente $27 \%$ de l'information et la seconde (ordonné) $12 \%$.

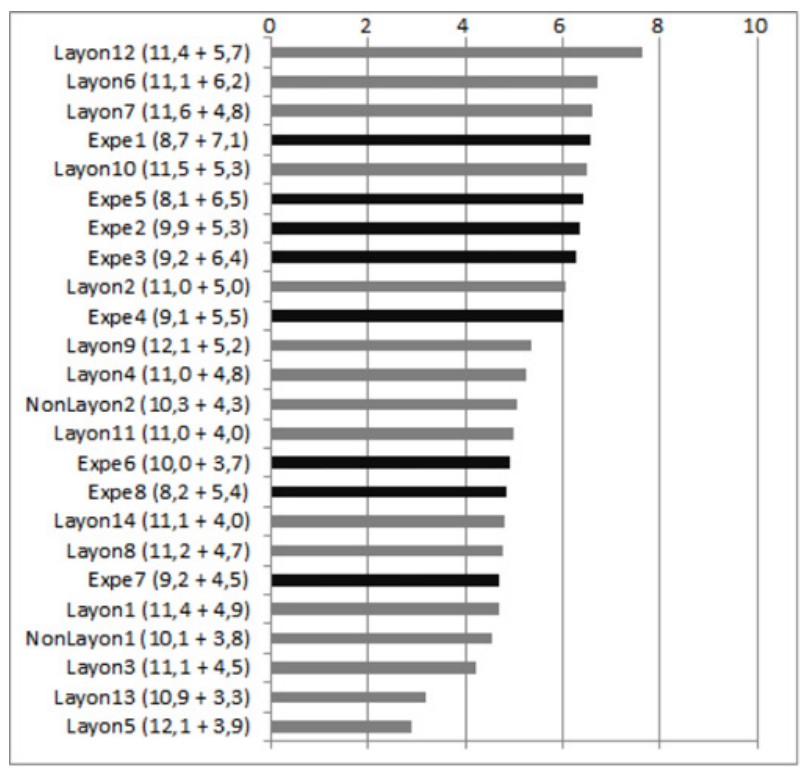

Figure 4. Notes moyennes d'exemplarité et (TAV et taux de sucre en \%vol) par produit.

\subsection{La typicitéperceptuelle des vins de l'AOC Coteaux du Layon par les professionnels de la filière}

L'ACP, avec les juges comme variables, montre qu'il y avait un fort consensus entre les juges (positionnement des juges/variables dans le même demi-cadran) (Fig. 3). Cela nous amène à confirmer qu'il existerait bien une typicité sensorielle des vins de l'AOC Coteaux du Layon, même si cette appellation a une certaine ancienneté et présente une certaine diversité.

La moyenne générale des notes de typicité obtenue est de 5,4 $\pm 0,70$. Deux paliers sont visibles sur la Fig. 5 : un premier après le vin Layon 13 et un second après le vin Layon9. Bien que ne respectant pas le cahier des charges de l'AOC, des vins expérimentaux ont des notes d'exemplarité supérieures à la moyenne de l'échelle et peuvent donc être considérés comme de bons exemples. Les vins expérimentaux Expe1 et Expe5 sont proches des vins avec les plus fortes notes moyennes d'exemplarité. Ce résultat montre que des vins à TAV faible peuvent avoir

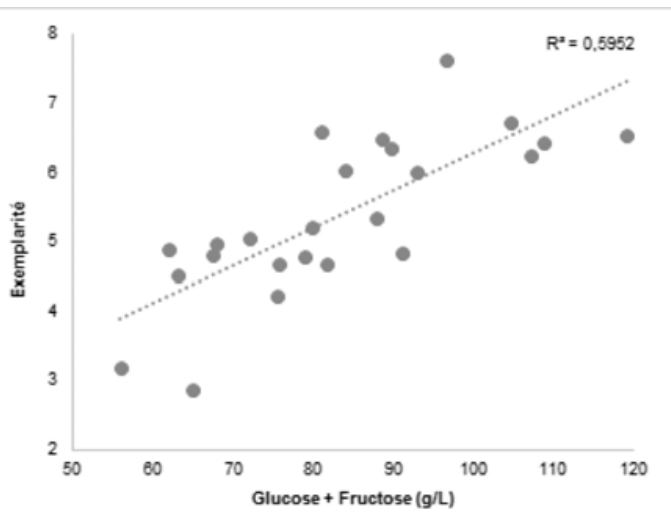

Figure 5. Relation entre les notes d'exemplarité et les quantités de sucres résiduels.

les caractéristiques sensorielles correspondant à la typicité sensorielle attendue des vins de l'AOC Coteaux du Layon.

Parmi les vins les plus typiques, plusieurs vins sont des vins décrits comme des vins aux arômes de «miel», de «fruits exotiques», «sucrés», et aux odeurs de «fruits blancs» selon le panel (Fig. 2). Ces caractéristiques correspondent à celles liées à la typicité conceptuelle.

La note d'exemplarité est fortement liée à la quantité de sucres résiduels (Fig. 4). Alors que le TAV semble, lui, jouer un rôle moindre. Ce résultat montre que les professionnels ont tendance à privilégier le sucre comme facteur de l'exemplarité. Ceci explique également pourquoi les vins Expe6 et Expe7 ont des notes d'exemplarité inférieures aux autres vins expérimentaux, tous deux ont, en comparaison, peu de sucres résiduels par rapport aux autres produits expérimentaux.

Les vins Expe1 et Expe5 sont des vins jugés plus majoritairement que les autres vins comme de «très bons exemples» de l'AOC Coteaux du Layon (Figs. 4 et 7). Les professionnels ont décrit le vin Expe1 comme étant un vin «aromatique» et «concentré», et le vin Expe5 comme un vin «frais» et «équilibré». Le vin Expe5 a cependant était jugé comme étant trop «sucré». Le vin Expe8 est un vin noté comme étant un «bon exemple» par les professionnels et comme étant «frais» et «équilibré»; le vin Expe7 comme un «moins bon exemple» car pas assez «acide». Les vins Layon2 et NonLayon1 ont, eux, été jugés comme de «mauvais exemples». Le vin NonLayon1 est décrit comme étant «plat», «acide» et pas assez «sucré» et le vin Layon2 comme ayant une bonne odeur mais «soufré» et «évolué». Le produit Layon2 crée des désaccords entre professionnels. Il regroupe, en effet, le troisième plus grand nombre de «très bon exemple» après les produits Expe1 et Expe5, mais est aussi cité le plus grand nombre de fois comme «mauvais exemple». Le terme «évolué» a d'ailleurs été utilisé en terme positif ou négatif.

\subsection{L'acceptabilitépar les consommateurs des vins avec de nouveaux profils analytiques}

Les consommateurs ont d'une manière générale apprécié les vins qui leurs étaient proposés, toutes les moyennes sont supérieures à 5 (Fig. 7). De plus, tous les vins ont été notés comme étant «agréables» par plus de $50 \%$ des consommateurs. Les trois vins les plus appréciés par les consommateurs sont : Expe1, Expe5 et Expe8, avec plus 


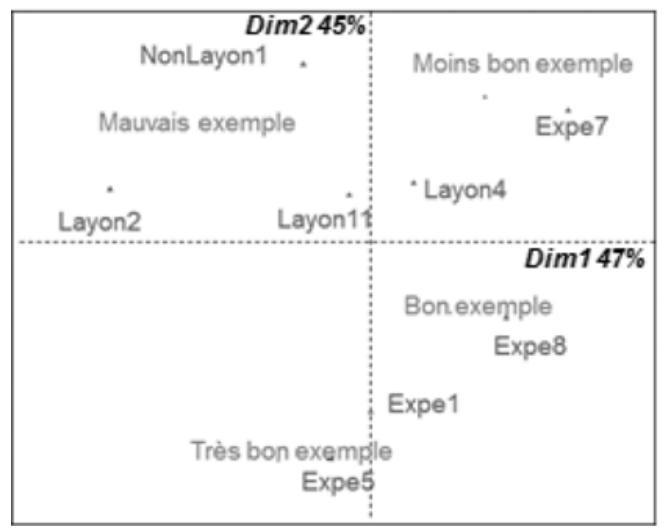

Figure 6. Analyse Factorielle des Correspondances sur la séparation des produits par les professionnels selon leur niveau d'exemplarité. La première dimension (abscisse) représente $47 \%$ de l'information et la seconde (ordonné) $45 \%$.

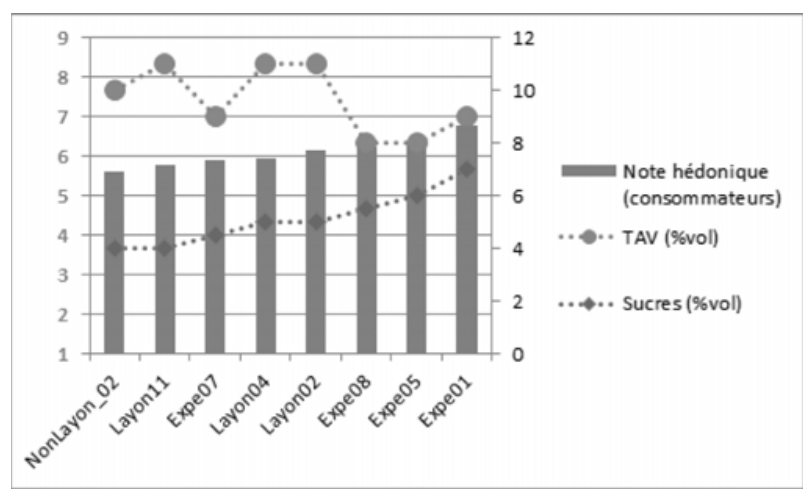

Figure 7. Moyenne de l'appréciation des consommateurs pour chaque vin et niveaux des Titres d'Alcool Volumique (TAV en $\%$ vol.) et des quantités de sucres résiduels (exprimées en \%vol).

de $85 \%$ des consommateurs qui ont considéré le vin Expe1 comme agréable. Ces trois vins sont ceux ayant le plus de sucres résiduels. Cette corrélation positive entre la moyenne obtenue et le taux de sucre résiduels est d'ailleurs confirmé Fig. 7. Le taux d'alcool semble, lui, ne pas jouer de rôle dans la notation des produits par les consommateurs.

\section{Conclusion}

Le cahier des charges d'une AOC permet de protéger le savoir-faire des vignerons d'un territoire. Une évolution du cahier des charges ne doit pas amener à modifier le produit. L'aspect innovant de la démarche était d'intégrer l'appréciation du consommateur à l'étude de la typicité mais aussi de coupler des dégustations individuelles, avec les professionnels, pour évaluer et caractériser la typicité, comme proposé par Cadot et al. [3], avec des tables-rondes pour pouvoir échanger sur les résultats et avoir alors des commentaires complémentaires.

Les résultats montrent qu'en considérant un TAV minimal de $10 \%$ vol (soit $-1 \%$ vol. par rapport au seuil actuel), des équilibres «alcool/sucre» de $10+4$ ou $10+5$ sont des équilibres qui semblent conformes à la typicité de l'AOC Coteaux du Layon et à l'appréciation des consommateurs. L'étude montre également que la composante «sucré», et sans doute les équilibres «acide-sucre», ainsi que la composante aromatique sont probablement plus importants pour la construction de l'exemplarité des vins de l'AOC Coteaux du layon que la seule quantité d'alcool présente au final dans le vin. D'ailleurs, il semble également qu'une majorité de consommateurs ne soit pas très sensible à cette question de la quantité d'alcool dans ce type de vins.

La notion d'équilibre «sucre/acide» a été évoquée lors des 3 tables-rondes. Les résultats mettent en avant l'importance de rester avec une quantité de sucre résiduelle assez importante.

Les résultats de l'étude vont permettent aux vignerons du syndicat des Coteaux du Layon en concertation avec la fédération viticole Anjou-Saumur, d'avoir des éléments factuels sur lesquels se reposer pour envisager de faire une demande d'actualisation du cahier des charges en faveur d'un abaissement du TAV minimum, auprès de l'INAO.

Une étude complémentaire sur la définition d'itinéraires techniques permettant d'obtenir et de reproduire des vins avec ces nouveaux équilibres peut aussi être envisagée. En perspectives, il serait aussi pertinent de focaliser une étude sur la notion d'équilibre avec un plan d'expérimentation adapté pour étudier cette question (ce n'était pas l'objectif ici).

Ces travaux ont reçu le soutien financier d'Interloire et du ministère chargé de l'agriculture (CASDAR). La responsabilité du ministère chargé de l'agriculture ne saurait être engagée.

\section{Références}

[1] http://www.vinetsociete.fr/mediatheque\# publications, consulté le 5 avril 2016

[2] P. Colonna, S. Fournier, J.-M Touzard, Chapitre 4. Système alimentaire. Durabilité de l'alimentation face à de nouveaux enjeux. Questions à la recherche (INRA-CIRAD, 2011)

[3] Y. Cadot, S. Caillé, A. Samson, G. Barbeau, V. Cheynier Anal. Chim. Acta 660, 1-2 (2010)

[4] E. Neethling, G. Barbeau, C. Bonnefoy, H. Quénol, Climate Res 53 (2012)

[5] F. Sauvageot, Sci. aliments 14 (1994)

[6] F. Casabianca, B. Sylvander, Y. Noël, C. Beranger, J.-B. Coulon, G. Giraud, G. Flutet, F. Roncin, E. Vincent In VIth International Terroir Congress (2006)

[7] L. Perrin, J. Pages, J. Sens. Stud. 24, 5 (2009)

[8] J. Ballester, C. Dacremont, Y.L. Fur, P. Etiévant, Food Qual. Prefer. 16, 4 (2005)

[9] I. Maitre, R. Symoneaux, F. Jourjon, E. Mehinagic, Food Qual. Prefer. 21, 7 (2010)

[10] A. Loison-Bou Maroun, R. Symoneaux, P. Deneulin, T. Thomas-Danguin, C. Fant, L. Guérin, Y. Le Fur, Food Qual. Prefer 40, A (2015) 